\title{
An Adaptative Multi-Agent System Approach for Image Segmentation
}

\author{
Redjimi Mohammed \\ Phd, Department of Computer Sciences University \\ of 20 August 1955- Skikda- \\ Algeria
}

\author{
Amri Said \\ Department of Computer Sciences University of \\ M'sila- Algeria
}

\begin{abstract}
This article presents a multi-agent approach for the segmentation of images. A multi-agent system (MAS) is a distributed system consisting of a set of agents that interact with themselves in an environment they are able to perceive and on which they can act. The proposed solution consists in cutting the space of the image to treat it in a set of sub-spaces (partitions of the image) in which several agents are created to detect the outlines of objects then to follow them (these agents are called detector - followers agents). These agents adapt a very efficient algorithm of detection and follow the outline according to the characteristics of the region that they evolve in. The information so collected is transmitted to levels of supervision agents (agents partitions) which take care they with collecting the information emitted by the agents detector - followers, to update tables containing the parameters of segmentation and to elaborate global strategies of management of the agents detector - followers (creation, destruction, setting in a stand-by mode or initialization of agents detector-followers). At the highest level of this agent's hierarchy, we find the supervisor agent of this whole system. An implementation of this approach by the use of Madkit system allowed us to observe a gain in performances and in precision very important due to parallel, concurrent and cooperating execution of tasks.
\end{abstract}

\section{General Terms}

Image processing

\section{Keywords}

Agent, multi-agent system (MAS), outline detection, image segmentation

\section{INTRODUCTION}

The segmentation of images is a very important step in the process of computer vision. Its objective is to provide a compact and convenient formal description of objects contained in the image by the extraction of various visual indications, mainly the regions as well as the outlines of objects. Several researches were led with the aim of determining the optimal methods for the segmentation of images.

Review of literature shows that there are several possible methods of segmenting an image. The best method is the one that allows a good understanding of the image [1][2] [3][4][5]. Generally, a real image contains a set of regions with different characteristics according to criteria of noise, fuzziness and neatness. It is then relevant to adopt an adaptive approach of detection of outlines operating in homogeneous regions in the sense of these criteria [4].

The performance of a detector is strictly proportionnal to the time of calculation and to the efficiency of the detection. The efficiency of detectors can be defined according to the following three criteria : [1]

- Detection of all outlines: all the outlines must be detected (without omission of certain pixels on the outline to be detected)

-Localization of the outlines detected in their ideal position.

- Detector does not have to supply with multiple answers or with false outlines.

Agent-based approaches are bio-inspired approaches which provide a solution and several advantages such as autonomy, pro-activity, distribution, self-organization and adaptation. The agents cooperate for collective problem solving. An isolated agent cannot solve the entire problem because it has not large perception and capabilities. So, the system of agents provides a collective solution that emerges from collective actions within the population of the agents [6],[7],[8].

We present a work using MAS for image segmentation. Our solution consists in cutting the space of the multi-agent system work according to three hierarchical levels:

- At the low level of the image (the level of pixels): a set of reactive agents detects then follow the outlines of objects contained in an image partition (these agents are called detector-followers agents).

- At an intermediate level, agents (called partition agents) oversee the activities of the detector-followers agents and perform many management tasks concerning these agents, receive the data concerning outlines detected by the agents following detectors and update tables for their management.

- At the high level, a master agent supervises all the system. It performs several tasks: after receiving a numerical image from the input, it divides the image in $\mathrm{k}$ partitions according to its complexity (the more the image is complex, the bigger will be the number of partitions). Then, the system creates an agent by a partition (agent partition) to whom it confers the detection of the outline of this one. At the end of the process of detection, it creates a global table containing the totality of outlines so obtained.

The agents communicate between them for the information exchange according to simple and not ambiguous protocols. We adopt adaptive techniques for detection and follow-up of outlines (The adopted filters are selected according to the characteristics of regions: net, fuzzy or noise).

The proposed approach was implemented on the platform multi-agents MadKit (Multi-Agents Development Kit) [9] to benefit from the possibilities offered by this software tool. The obtained results are very satisfactory and show the efficiency of the approach. This article is organized as follows: Section 2 is dedicated to the main approaches of use of the multi agent systems in artificial vision. Our approach of segmentation is presented in section 3. Section 4 illustrates some methods that we have used in our work. Section 5 
presents some results realized on the platform Madkit. Section 6 is devoted for the conclusion of the work and for its perspectives.

\section{RELATED WORKS}

For several years, a particular interest was granted in the use of multi agent systems for the resolution of problems in artificial vision. Several works propose good solutions to deal with various open issues, mainly in image segmentation by the distribution of the parallel processing.

F.Bellet[10] presents incremental processes of region growing or outlines. The cooperation between two types of agent is dynamic and allows to transmit Information when it becomes necessary for a taking of decisions.

In the same order of idea Boucher [11] suggests using specialized agents for the segmentation and the interpretation of sequences of cytological images. It is a distributed approach where every agent is specialized in recognition of a concept of the image.

P. Remagnino and al [12] used a multi-agents system that we consider as cognitive for the supervision of dynamic scenes. The authors associate for every object of the analyzed scene an agent. The system uses a Bayesian network to deduce one semantic of the movements of the various objects. For every object in the scene, an agent of behavior is created to operate at the level object. The reasoning of this agent is made on the localization, the speed, the acceleration and the trajectory of the object.

The multi-agent system proposed by Ramos and Almeida [13] is a typical system based on the reactive approach. Based on the colonies of artificial ants, developed initially by Chialvo and Millonous [ 14 ], the system proceeds to the segmentation of an image at levels of grey, by considering it as the environment in which move ants. The authors showed that collective perceptive capacities emerge from local interactions between the various agents on one hand, and between the agents and the environment of somewhere else.

Liu and al. [15] created a reactive multi agent system for brain MRI segmentation. They affirm that employing agents are more efficient than the classical region-based algorithms. The pixels are labeled by using four types of agents according to their belonging to a region. The local perceptions of the agents infer their actions.

Richard and al. [16] introduce a hierarchical system architecture of cooperative agents for image segmentation of brain MRI. In this work, three types of agents, which operate at three control levels are used: global control agent, local control agent, and tissue dedicated agent. At the global control level, the agent is devoted to divide the volume of data into adjacent zones and to assign each zone to one local control agent. The tissue dedicated agents are created by local control agents and use the region growing approach. Parameters needed to perform this approach are updated according to the interaction between adjacent agents.

Mahsa Chitsaz and al. [17] [18] propose a method for medical image segmentation which uses the properties of agent in a MAS environment. The original image is partitioned into a set of sub-images. They used two hierarchical levels : Local level who each local agent works on a sub-image. It seeks to mark each pixel as a specific zone using of given a priori-knowledge. Global level who a monitoring (moderator) agent builds a final segmented image by receiving the results of all agents' work.

\section{PROPOSED SYSTEM}

The organization of the multi-agent system is articulated around three groups (Fig. 1):

- The group of masters: which performs high level processing, (This group contains one agent)

- The partition groups: intermediate level ( this group is composed of $\mathrm{k}$ agents)

- The group of detector-followers : low level processing (the agents here are created and deleted dynamically).

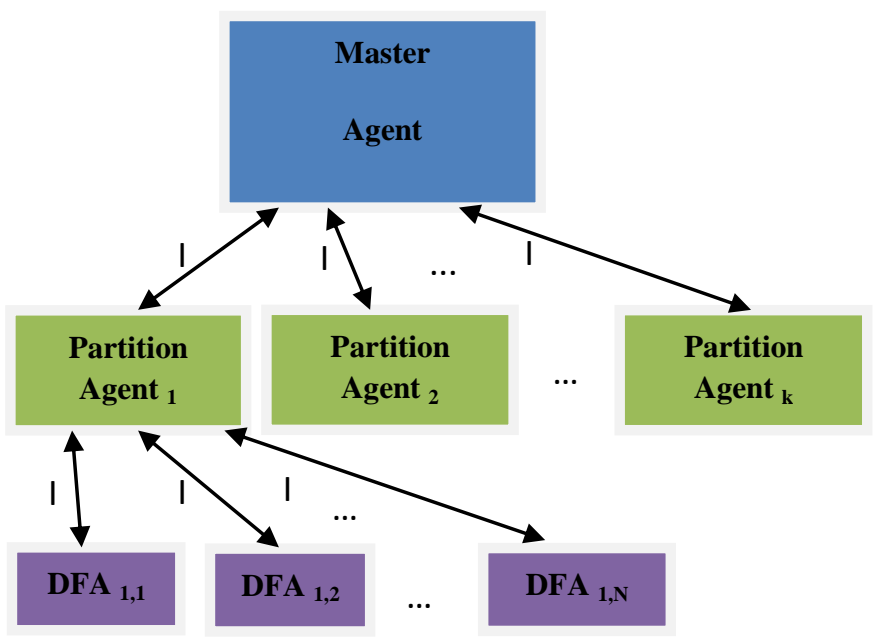

Legend :

- Master agent: monitoring agent.

- Partition agent ${ }_{\mathrm{i}}$ : Agent who is in charge of partition number i.

- $\quad$ DFA I,j : Agent detector-follower $\mathrm{j}$ in the partition $\mathrm{i}$

- I : Interactions between agents.

Fig. 1 : The multi-agent system

\subsection{Master agent}

The master agent performs high er level processing, it supervises all the system. It plays the following rules:

- $\quad$ Receive the digital image from an input device.

- Evaluate the complexity of the image in order to create a number of partitions.

- Create and initialize the partition agents.

- $\quad$ Put in the sleep or kill partition agents.

- Receive the data tables of outline from the partition agents.

- $\quad$ Rebuild the segmented image. 
- Stop the segmentation process at the end of the works.

\subsection{Partition agents}

It is a set of $\mathrm{k}$ agents, an agent per partition ( $\mathrm{k}$ is chosen according to the complexity of the image: more the image is dense, more $\mathrm{k}$ is big. However, in order to obtain good system's performance, the smallest partition of the image does not have to be smaller than $8 \times 8$ pixels and $\mathrm{k}$ smaller than 4 ). A partition agent plays the following rules:

- Label the regions inside the partition according to table 1 . This labeling is made according to the characteristics of each zone and permit to detector-followers agents to adapt the optimal detection-follow method.

- Create and initialize the detector-followers agents.

- emit the image partitions to the detector-followers agents.

- put in the sleep or killing detector-followers agents.

- receive the data relative to outlines detected and followed by the detector-followers agents.

- Update the tables of the partition.

- Send these tables to the master agent at the end of the work.

Therefore, a set of attributes characterizes a partition agent:

- $\quad$ APC: Agent partition code (in order to identify the partition)

- NDF: Number of detector-followers agents.

- TDO: Tables of detected outlines.

- TDS: Tables of detector-followers agents states.

- EP: state of the partition (1: if all the partition has been treated, else: 0 ).

A special word is utilized for acceding to these data (SWP).

\subsection{Detector-followers agents}

These agents work at the image's lower level in order to detect and follow the points of outlines. They use appropriate methods for the detection and follow of outline. The agent chooses his operator of segmentation according to the label of the zone where it is (see table 1). It means that agent adapts the algorithm according to the state of the region.

For example: if the label of the zone is 2 (a zone with levels of details), the agent chooses the operator of Sobel because this last one gives good results in this case.

The detector-following agents are in charge of: look for a point of outline, memorize this first point of outline, follow the outline corresponding to the detected point, and send the set of data corresponding of the outline detected to the partition agent. Mark every treated pixel crossed to make sure that another agent does not follow it a second time. At the meeting of the first point of outline; the agent Detectorfollower crosses in the stage follow-up of outline. Every Agent detector-follower uses the code of Freeman to follow the detected outline. This code allows to represent the outline of an object by means of a suite of digits between 0 and 7 , representing each a direction (Fig 2.a). The agent tests the directions as one goes along. If the pixel following is a point of the outline, it adds the value of direction in the list of codes of Freeman, then it passes in the direction following one. The agent continues until it falls again on the point of departure (Appear Fig2.b) or if it arrives on the frame of the partition or still if there is no point anymore following on eight directions of Freeman. Each pixel crossed is marked. A data sequence is memorized in an agent table which is transmitted to the partition agent at the end of the work of this agent.

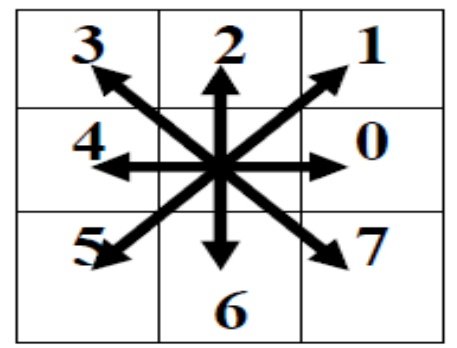

Fig. 2a: The eight directions of the Freeman code.

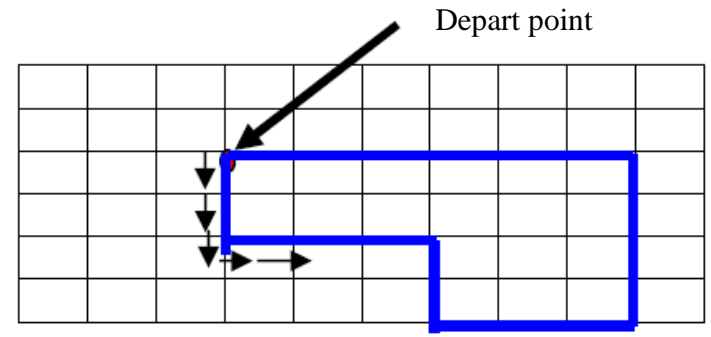

List of directions: $(i, j)(66000660002222444444)$

Fig.2 b: example of list of points.

An agent detector-Follower is characterized by a set of attributes:

- CS: current state (in exploration, in follow-up or in wait).

- CP: Current Position (x, y) in the partition.

-CADF: Code of detector-Follower agent (identifier)

This information constitutes the state word (SWDF) of the agent.

\section{CHOICE OF THE METHOD OF OUTLINE DETECTION}

Being given that an image can contain a set of zones with different characteristics regarding noise, fuzziness, and 
neatness, it is difficult to have one effective detection of outlines when we apply only one operator or only one leaks out of this image in its entirety. To improve the efficiency of the detection, each of the agents Detectors-followers use its own operator of detection According to criteria of noise, fuzziness or neatness associated with the zone that operate the agent. To be able to identify zones, we need first of all estimate the noise, the fuzziness and the neatness. [4][19][20]. Note: we present in this section some methods that we used in our work.

\subsection{Fuzzy estimation}

For estimation of the fuzziness, we use the Gaussian filter of the images.

If $I(x, y)$ is the original image of size NxM and is I '(x,y) the image filtered by a Gaussian, then, the estimation of fuzziness( Noted $E_{f}$ ) in equation 1, at the level of a zone (region PxQ) can be Formulated as follows: [4][19]

$\mathrm{E}_{\mathrm{f}}=\sqrt{\frac{\sum_{X=p, y=q}^{P+p-1, Q+q-1} I(x, y)^{2}}{\sum_{X=p, y=q}^{P+p-1, Q+q-1} e(x, y)^{2}}}$

$\mathrm{E}(\mathrm{x}, \mathrm{y})=\mathrm{I}(\mathrm{x}, \mathrm{y})-\mathrm{I}^{\prime}(\mathrm{x}, \mathrm{y})$ pixel difference between the two images at the $(x, y)$ position.P, $\mathrm{Q}$ coordinated initials of a zone of size pxq.The fuzzy presence is detected when $\mathrm{I}(\mathrm{x}, \mathrm{y})$ and $\mathrm{I}^{\prime}(\mathrm{x}, \mathrm{y})$ are similar and $\mathrm{E}_{\mathrm{f}}$ is big.

\subsection{Noise estimation}

The noise estimation is calculated from the variance of the derived image signal :

$\mathrm{d}=\frac{\sum_{x=p}^{P+p-1} \sum_{y=q}^{Q+q-1} D(x, y)}{P . Q}$

$\boldsymbol{\sigma}_{\mathrm{z}}{ }^{2}=\frac{\mathbf{1}}{(\mathrm{d}+255)^{2}} \frac{\sum_{x=p}^{P+p-1} \sum_{y=q}^{Q+q-1}(D(x, y)-\mathrm{d})^{2}}{\boldsymbol{P} \cdot \boldsymbol{Q}}$

d (equation 2) is the average of intensity of the pixels in the PxQ zone. If each pixel is coded by using 8 bits, 255 is the intensity maximum and $(\mathrm{d}+255)^{2}$ is a normalization coefficient. $\mathrm{D}(\mathrm{x}, \mathrm{y})$ is the pixel intensity of the derived image at the $(\mathrm{x}, \mathrm{y})$ position and $\sigma_{\mathrm{z}}{ }^{2}$ (equation 3 ) is the variance of a derived image of a zone of PxQ size.

Experimental results [4][19] show that, in the case of net images with level of details, the Sobel operator is better than Roberts, Prewitt or Kirsch. In the case of noised image, MIRA [4][19] detector is the best. If we have an uniform image with little details the Mallat wavelet detector seem the most suited.

\subsection{Choosing a detection operator}

M. Sarifuddin, R. Missaoui et H. Laggoune [19] develop a news filter called MIRA (Multimedia Information Retrieval Application) based on an outline fuzzy detection. The first (equation 4) aims to attenuate the noise and the second (equation 5) is destined for outline detection.

$$
\begin{aligned}
& \mathrm{F}(\mathrm{x})=\operatorname{sgn}(-\mathrm{x}) \mathrm{k}_{1} e^{-\alpha|x|}\left[1-\cos (\beta|\mathrm{x}|)+\left[\frac{\alpha^{2}-\beta^{2}}{2 \alpha \beta}\right] \sin (\beta|\mathrm{x}|]\right. \\
& \mathrm{H}(\mathrm{x})=\mathrm{k} \cdot e^{-\alpha|x|}\left[2-\cos \left(\beta|x|+\frac{\alpha}{\beta} \sin (\beta|x|]\right.\right.
\end{aligned}
$$

$\beta$ Fuzzy parameter $\alpha$ Noise parameter $0<\beta<\alpha \sqrt{\pi} / 4$

\begin{tabular}{|c|c|c|c|c|}
\hline $\begin{array}{c}\begin{array}{c}\text { Fuzzy } \\
\text { estimator }\end{array} \\
\mathbf{E}_{\mathbf{f}}\end{array}$ & $\begin{array}{c}\text { Noise } \\
\text { estimator } \\
\sigma_{\mathrm{z}}{ }^{2}\end{array}$ & $\begin{array}{l}\text { Type of } \\
\text { region }\end{array}$ & $\begin{array}{l}\text { Use } \\
\text { operator }\end{array}$ & Label \\
\hline$<50$ & $>0.1$ & Fuzzy & $\begin{array}{c}\text { MIRA Filter } \\
0.25<\alpha<1 \\
\text { and } \\
|\beta|=\alpha / 1.125\end{array}$ & 0 \\
\hline $\begin{array}{l}<25 \text { or } \\
>800\end{array}$ & $<0.002$ & $\begin{array}{c}\text { Net } \\
\text { uniform } \\
\text { or } \\
\text { pseudo } \\
\text { uniform }\end{array}$ & $\begin{array}{c}\text { Mallat } \\
\text { wavelet }\end{array}$ & 1 \\
\hline$<50$ & $\begin{array}{c}>0.002 \\
\text { and } \\
<0.075\end{array}$ & $\begin{array}{c}\text { Net with } \\
\text { levels of } \\
\text { details }\end{array}$ & SOBEL & 2 \\
\hline $\begin{array}{c}>50 \text { and } \\
<800\end{array}$ & $<0.075$ & Fuzzy & $\begin{array}{l}\text { MIRA } \\
0.2<|\beta|<0.8 \\
\alpha=\beta^{*} 1.125\end{array}$ & 3 \\
\hline$<50$ & $\begin{array}{c}>0.075 \\
\text { and }<0.1\end{array}$ & $\begin{array}{c}\text { Net with } \\
\text { levels of } \\
\text { details }\end{array}$ & $\begin{array}{l}\text { MIRA } \\
\alpha \geq 1 \text { and } \\
|\beta|=0.2\end{array}$ & 4 \\
\hline
\end{tabular}

Table 1. Labeling type of regions.

\section{IMPLEMENTATION AND EXPERIMENTAL RESULTS}

We used the methodology AALAADIN for the design of this work. Our Multi-agents system was implemented by using the platform Madkit. This last one constitutes a powerful software tool for the development of this kind of systems and allows thousands of agents to work all independently in parallel. We used these two tools for their simplicity of implementation as well as for their power. They offer several mechanisms of development and implementation of applications. They are tools with academic impact. MADKIT platform can be free downloaded (http://www.madkit.org)

MADKIT use the Agent Group Rule (AGR) (Figure 3) who an agent plays one or more roles in one or several groups, therefore we have designed three groups of agents: Master group which concern the master agent who it play rules 
consisting in the tasks it performs (such as partitioning image, creating partition agents, etc.). The second group concerns the partition agents and it is composed of the $\mathrm{k}$ partition agents who they play the rules such as: to initialize detectorfollowers agents, to estimate characteristics of regions in their partition, etc.The last group includes the detector-followers agents and their rules are the tasks that they perform.

The information exchange between agents is vertically organized and it is realized according to message passage allowed in MADKIT.

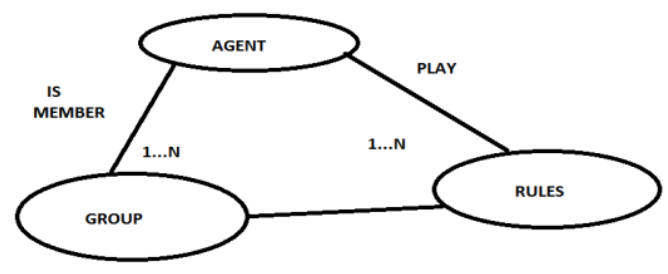

Fig. 3: The AGR Model.

The algorithms of detection and of detection follow-up of the outlines have been programmed by using the environment of programming MATLAB Version 7.8.0.347. Finally several components JAVA have been used (multi-agent platform MADKIT is written in JAVA).

Figure 4. shows some examples of application of our method in the segmentation of images then their reconstruction. We present numerous type of images (a,b: electronic circuit; c,d: medicine ;e,f: face ; h,g: text) We see clearly that outlines were followed well. The images resolution is one mega pixels(1024x1024). The number of partitions of the image is different as it is about complex images (examples: (a) and (h) include many details for whom a large number of partitions were created). For the images (a) and (h), the system creates partitions of $8 \times 8$ pixels, or of simple images containing few details. The applied filters are different as we are in a fuzzy, noisy or net zones. Examples (c) and (e) show these three notions. Within every partition, there is a random creation of detector-following agents. In fact, at the beginning a single agent is creating, this agent looks of a point of outline. When it finds this point, the agent marks and memorizes its coordinates and follows the outline (the suite of the point are memorized in the table of the agent according to Freeman code). At the same moment (moment of the detection), a second agent is created in the line which follows ( $i+1)$ and the same process is initialized. To avoid to overlapping which would give; in fact; much more robustness to our system but it is risks to end in an endless, any crossed pixel is marked. We have experimented our system by using a database of more than three thousand images with many of the differences (we have voluntary incrusted many details in the original images With the aim of testing the performances of our system; like Salt-and-pepper for creating of noises, levels of details in images like ULSI architecture images, satellites images, etc.). We noticed that our system gives satisfactory results in precision and in gain in times of execution.

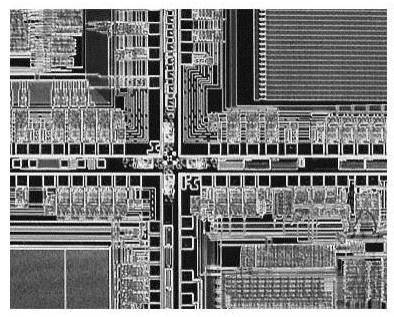

a
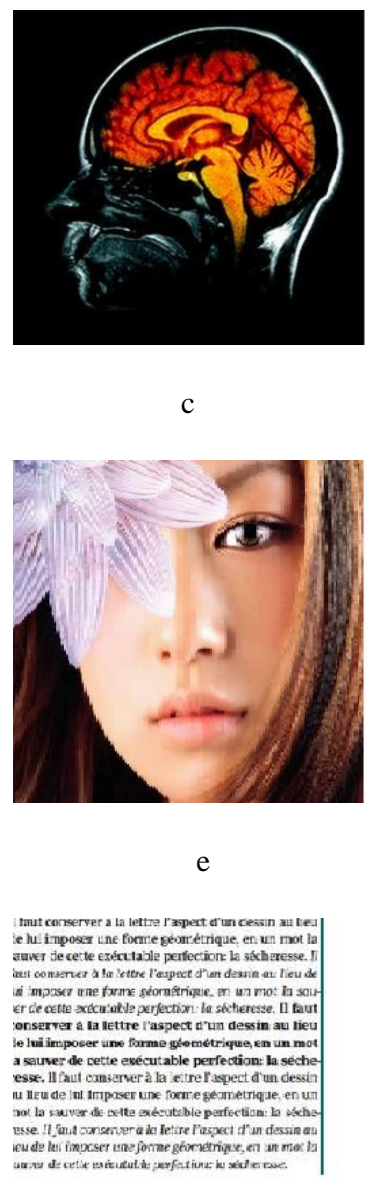

$\mathrm{h}$

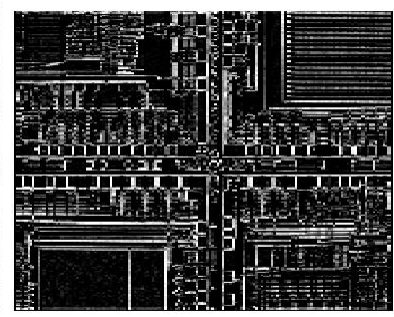

b

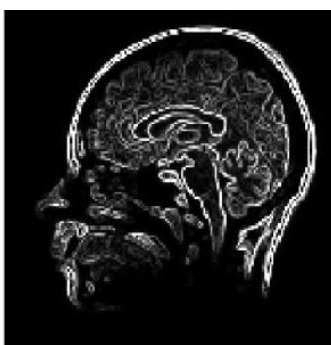

d
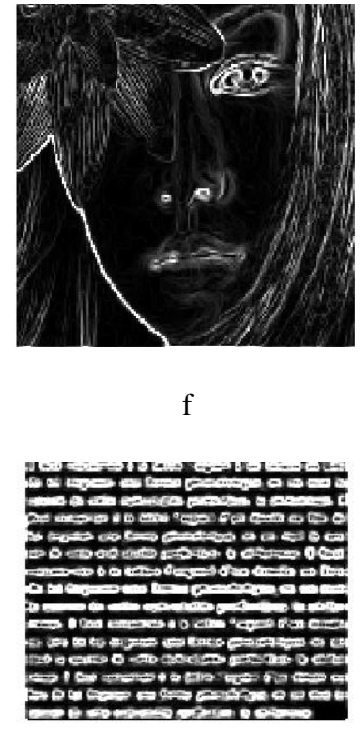

Fig 4. Examples of outlines results

\section{CONCLUSION}

The obtained results are very satisfactory, and demonstrate the adaptation of our agents of segmentation in front of a big variety of images. Our approach has big advantages with regard to other techniques, thanks to the automatic choice of the method of detection by the detector-followers agents characteristics of their zones. Every detector agent adapts the most effective algorithm of detection according to the characteristics of his region.For future perspectives, we think extend our work for including a recognition system of images based around MAS architecture. 


\section{REFERENCES}

[1] Canny, J. November 1986. A Computational Approach To Edge Detection. IEEE Trans. Pattern Analysis and Machine Intelligence, 8:679-714.

[2] Marr, D. and Hildreth, E.C. 1980 Theory of Edge Detection. Proc. of the Roy. Soc. Lond. B, Vol 207.

[3] Shi, J. and Malik, J. August, 2000 Normalized Cuts and Image Segmentation. IEEE Transactions on Pattern Analysis and machine Intelligence , 22(8), pp. 888-905.

[4] Missaoui, R. Sarifuddin, M. , Baaziz, N. and Guesdon, V. March 2005 Détection efficace de contours par décomposition de l'image en régions homogènes. Actes de la conférence SETIT 2005, Sousse-Tunisie. (In french)

[5] Fan, T.J., Medioni G.G., and Nevatia., R. December 1987 Segmented description of 3-D surfaces. IEEE J. Robotics Automat., 3(6):527-538.

[6] Ferber, J. 1999 Multi-Agent Systems: An Introduction to Distributed Artificial Intelligence Addison-Wesley Longman Publishing Co., Inc., Boston, MA, USA.

[7] Krishnamurthy, E. V. and Murthy, V.K. 2006 Distributed agent paradigm for soft and hard computation. Journal of Network and Computer Applications, 29(2):124-146

[8] Ferber, J. 1995 Les systems multi-agents vers une intelligence collective. Interedition Paris. (In French)

[9] Gutknecht, O. and Ferber J. 2000 The MadKit agent plateforme architecture. Laboratoire d'Informatique, Robotique et Microélectronique de Montpellier

[10] Bellet, F. June 1998 Une approche incrémentale, coopérative et adaptative pour la segmentation des images en niveau de gris. Institut National Polytechnique de Grenoble, France. (In french)

[11] Boucher, A. Fevrier 1999 Une approche décentralisée et adaptative de la gestion d'informations en vision. Thèse de doctorat de l'université Joseph Fourier Grenoble I. (In french)

[12] P. Remagnino, P. , Tan, T. and Baker, K. 1998 Multiagent visual surveillance of dynamic scenes. Image and vision computing, Elsevier Ed., vol. 16, pp 529-532.

[13] Ramos, V. and Almeida, F. 2000 Artificial Ant Colonies in Digital Image abitats. in: Dorigo, M. et al (editors), Proceedings of ANTS'2000 - 2nd International Workshop on Ant Algorithms pp 113-116,Brussels, Belgium.
[14] Chialvo, D. and Millonas, M. 1995 How Swarms Build Cognitive Maps. in: L. Steels (editor), The Biology and Technology of Intelligent Autonomous Agents, pp 439. 450, NATO ASI Series.

[15] Liu, J. and Tang, Y.Y. June 1999 Adaptive Image Segmentation With Distributed Behavior-Based Agents. IEEE Trans. Pattern Analysis and Machine Intelligence, vol. 21 , no. 6 , pp.544-551

[16] Richard, N. , Dojat, M. and Garbay, C. 2004 Automated segmentation of human brain MR images using a multi-agent approach. Artificial Intelligence in Medicine, 30(2):153-176.

[17] Chitsaz M. and Seng W.C. 2009 Medical Image Segmentation by Using Reinforcement Learning Agent. icdip, pp.216-219, 2009 International Conference on digital Image Processing.

[18] Chitsaz, M. and Woo, C S. 2008 The rise of multiagent and R.L. segmentation methods for biomedical images. In Proc. The 4th Malaysian Software Engineering Conference (MySEC 2008), Kuala Terengganu, Malaysia.

[19] Sarifuddin, M. , Missaoui R. and Laggoune, H. March 2005 Technique de détection de contours flous et bruités. Actes de la conférence SETIT 2005, SousseTunisie. (In french)

[20] Bourennane, E., Gouton, P. , Paindavoine M. and Truchetet, F. 2002 Generalization of Canny-Deriche Filter for detection of noisy exponential edge. Signal Processing 82 pp. 1317 - 1328 .

\section{AUTHOR'S PROFILE}

Dr.Redjimi Mohammed received the Diploma of Docteuringenieur in computer sciences from the University of LILLE 1 - FRANCE (1984) and the post-doctoral Degree Habilitation Universitaire from the University of ANNABA ALGERIA (2007). He is currently an associate professor of computer science at the SKIKDA University (ALGERIA). His main research interests include modeling and simulation systems mainly by using multi-agents systems, image recognition and computing hardware and software systems.

Amri Said received the Diploma of Magister in computer science from the University of KHENCHELA- ALGERIA (2009). He is currently, Maitre assistant at M'SILA University (ALGERIA). He prepares a Doctor thesis in computer science on the collaborative approaches for the medical images segmentation supervised by $\mathrm{Dr}$ Redjimi Mohammed. 